\title{
Tendency to adhere to provider-recommended treatments and subsequent pain severity among individuals with cancer
}

This article was published in the following Dove Press journal:

Patient Preference and Adherence

14 January 2011

Number of times this article has been viewed

\author{
Anthony Jerant ${ }^{\prime}$ \\ Peter Franks' \\ Daniel J Tancredi² \\ Naomi Saito ${ }^{3}$ \\ Richard L Kravitz ${ }^{4}$ \\ 'Department of Family and \\ Community Medicine, Center for \\ Healthcare Policy and Research, \\ University of California Davis School \\ of Medicine, Sacramento, CA, USA; \\ ${ }^{2}$ Department of Pediatrics, Center \\ for Healthcare Policy and Research, \\ University of California Davis School \\ of Medicine, Sacramento, CA, USA; \\ ${ }^{3}$ Center for Healthcare Policy and \\ Research, University of California \\ Davis School of Medicine, Sacramento, \\ CA, USA; ${ }^{4}$ Department of Internal \\ Medicine, Division of General \\ Medicine, Center for Healthcare \\ Policy and Research, University of \\ California Davis School of Medicine, \\ Sacramento, CA, USA
}

Background: Patients' general tendency to adhere to health care provider-recommended treatments is associated with a number of health outcomes, but whether it influences pain severity over time among individuals with cancer is unclear. We explored the relationship between adherence tendency and subsequent pain severity among cancer patients participating in a randomized controlled trial of coaching to enhance communication with physicians and reduce pain severity.

Methods: Associations between baseline Medical Outcomes Study general adherence measure score and pain severity over 12 weeks were examined with repeated-measures regression models. Model 1 included sociodemographics, study group and site, follow-up point, and baseline pain; Model 2 included these variables plus partner status, physical and mental health status (12-item Short Form Health Survey [SF-12 $\left.{ }^{\circledR}\right]$ ), and pain control self-efficacy.

Results: Of 307 patients randomized, 224 (73\%) had at least one follow-up pain severity assessment plus complete data for other model variables and were included in the analyses. In Model 1, adherence tendency was associated with less subsequent pain severity: a one standard deviation increase in adherence tendency was associated with a 0.22 -point adjusted mean decrease in pain severity on a $0-10$ scale $(95 \%$ confidence interval $0.40,0.03)$. The association was diminished and not statistically significant in Model 2, primarily due to adjustment for the SF-12.

Conclusion: Tendency to adhere to provider-recommended treatments was associated with subsequent pain severity among individuals with cancer, suggesting a potential way of predicting and intervening to improve cancer pain control. However, the association was attenuated after adjusting for health status, suggesting mediation or confounding of the relationship by health status.

Keywords: health status, longitudinal studies, neoplasms, outcome assessment (health care)

Suboptimal pain control is common among individuals with cancer. ${ }^{1,2}$ However, whether a patient's general tendency to adhere to health care provider-recommended treatments influences cancer pain severity over time is unclear, as no studies have examined this issue. This is an important research gap for several reasons. Data from cross-sectional studies suggest low adherence to both scheduled and "as needed" analgesics prescribed for cancer pain. ${ }^{3,4}$ Although there appear to be multiple contributors to this problem, ${ }^{1,5}$ patient concerns regarding the potential side effects (eg, lethargy) and risks (eg, addiction) of analgesics prescribed for cancer pain are likely to play a role. Such concerns appear to be prevalent in society ${ }^{1,2,6,7}$ and, among those endorsing them, might lessen their tendency to adhere to recommended analgesic regimens. 
Low adherence to analgesics would, in turn, be expected to contribute to suboptimal cancer pain control, given that poor treatment adherence has been associated with worse outcomes in numerous studies involving health conditions other than cancer pain. ${ }^{8}$ Taken together, these observations suggest the importance of studying the relationship between patient tendency to adhere and subsequent cancer pain control. Additionally, although the phrase "tendency to adhere" may imply a largely fixed dispositional characteristic, prior intervention studies involving health conditions other than cancer pain have established that tendency to adhere can be favorably influenced, ${ }^{9}$ so may have both "trait" and "state" components. Thus, if a greater tendency to adhere was associated with reduced cancer pain severity over time, it might be a useful target for interventions aimed at improving cancer pain control.

To begin to examine these issues, we conducted secondary prospective observational analyses of data from Cancer Health Empowerment for Living without Pain (Ca-HELP), a randomized controlled trial (RCT) of a patient coaching intervention, designed to enhance patient communication with cancer physicians and help them cope with and manage cancer-related pain. ${ }^{10}$ The coaching intervention led to more active pain-related communication by patients ${ }^{11}$ but no improvement in the general tendency to adhere to providerrecommended treatments, as measured with the well validated Medical Outcomes Study (MOS) general adherence self-report measure, ${ }^{12,13}$ and no decrease in subsequent pain (unpublished data). In the current analyses, we examined the relationship between a general tendency to adhere to provider-recommended treatments at baseline and cancer pain severity at 2, 6, and 12 weeks after the baseline assessment. Based on the preponderance of prior evidence from studies involving patients with health conditions other than cancer, ${ }^{8}$ we hypothesized that a higher general tendency to adhere to recommended treatments at baseline would independently predict lower cancer pain severity over time.

We examined two main models, sequentially adjusting for different groupings of covariates that have been associated with pain ${ }^{14-21}$ and adherence ${ }^{22-36}$ in prior studies. Model 1 included baseline pain severity, basic sociodemographic characteristics (age, gender, race/ethnicity, and education) as well as RCT-related variables that might confound the adherence tendencies/pain relationship (study group, study site, pain follow-up time point). Model 2 included all Model 1 variables plus characteristics that might influence adherence tendency, pain, or both: partner status, cancer type and degree of spread, physical and mental health status, and pain control self-efficacy. We also conducted a cross-lagged regression analysis (regressing baseline pain severity on to adherence tendency measured at 6 week follow-up, regressing 6 week pain severity on baseline adherence tendency and then comparing the two resulting cross-lagged regression coefficients for adherence) in order to assess the directionality of the relationship between tendency to adhere and pain severity. Finally, we examined the effects of individually removing these variables from Model 2, to facilitate exploration of which might most strongly influence the association between tendency to adhere and pain severity.

\section{Methods}

Study activities were conducted from November 2006 to December 2008. The Institutional Review Boards affiliated with the three participating institutions granted approval for the study. The trial was registered at ClinicalTrials.gov (identifier NCT00283166). The funder (American Cancer Society) had no role in the collection, analysis, or interpretation of data.

In the parent RCT, tailored education and coaching were compared with enhanced usual care. Full details of the study methods have been published elsewhere. ${ }^{10} \mathrm{Here}$, we focus on methodological issues relevant to the current observational analyses.

\section{Patient recruitment, enrollment, and randomization}

Oncology physicians $(N=49)$ were recruited from three health care delivery systems in the greater Sacramento, California, USA, area: the University of California Davis, Kaiser-Permanente, and the Northern California Veterans Administration. Potentially eligible patients, identified from each practice using computer-generated lists, were mailed a study invitation letter along with a postage-paid postcard allowing them to opt out of the study if desired. All patients who had not returned the postcard within 3 weeks were contacted by phone, screened for eligibility, and invited to participate. Initial patient eligibility criteria were age $18-80$ years, intact cognition, able to speak English, and having one of eight cancer types (lung, breast, prostate, head and neck, esophageal, colorectal, bladder, and various gynecologic). To further determine potential eligibility, responding patients also answered two pain-related questions. The first question asked them to indicate their worst (eg, most severe) level of pain during the 2 weeks prior to assessment using a $0-10$ analog scale, with 0 representing no pain and 10 representing the worst pain imaginable. The second question asked them to indicate the degree to which pain had interfered with their usual daily activities over the 2 weeks prior to assessment on a 1-5-point Likert scale, 
with 1 representing no interference and 5 representing extreme interference. To be eligible for the study, participants had to have a response of 4 or greater to the first item, or a response of 3 or greater to the second item, or both. Patient exclusion criteria included major surgery scheduled within 6 weeks, enrollment in a hospice, being followed by a pain management service (beyond a single consultation), or inability to receive and/or complete mailed enrollment materials.

Patients meeting eligibility criteria and lacking exclusion criteria were enrolled and randomly assigned to tailored education and coaching or enhanced usual care. Enrolled patients were promised $\$ 40$ after completing the index study visit, and a second $\$ 40$ following completion of three scheduled follow-up data collection phone calls.

\section{Study visit procedures}

Patients were asked to arrive at their oncology office 1 hour prior to a scheduled appointment. On arrival, they were greeted by a trained health educator, who brought them to a quiet space, obtained written informed consent, and provided a pre-intervention questionnaire. The health educator then administered the patient's randomly assigned intervention, either tailored education and coaching intervention or enhanced usual care (control). ${ }^{10}$ Patients then completed a post-intervention, pre-visit questionnaire, attended their physician visit, and then completed a post-visit questionnaire. Follow-up questionnaires were administered via telephone at 2 weeks, 6 weeks, and 12 weeks after baseline.

\section{Measures}

\section{Adherence}

General tendency to adhere to provider-recommended treatments was assessed at the time of study enrollment and again at 6 week follow-up using the MOS general adherence measure. ${ }^{12,13}$ The measure has been used successfully in prior studies, ${ }^{13,33,37,38}$ including the Medical Outcomes Study for which it was developed, ${ }^{13}$ as have slightly reworded HIVand tuberculosis-specific versions. ${ }^{39,40}$ Scores on the measure have been significantly correlated with adherence to specific medications measured using a variety of approaches, including via electronic pill bottle monitoring devices. ${ }^{33,37,40}$ The five measure items as employed in the study are shown in Figure 1. A 6-point Likert response scale was used for all items, from $1=$ none of the time to $6=$ all of the time. Scores for individual items were averaged to yield a summary score ranging from 1 to 6 , with higher scores indicating better adherence. Cronbach's $\alpha$ for the measure in our sample was 0.80 at baseline and 0.89 at 6 weeks.

\section{Pain severity}

Pain severity was measured at study enrollment (the baseline value in our analyses), immediately pre-intervention, immediately post-intervention (but before the study oncologist visit), immediately post-visit, and at 2, 6, and 12 weeks. At each measurement point, pain severity was coded as the mean of average and worst reported pain over the prior 2 weeks, both assessed with $0-10$ analog scales, with 0 representing no pain and 10 representing the worst pain imaginable. ${ }^{10}$ Cronbach's $\alpha$ for the combined pain severity measure was $0.88,0.87,0.88,0.89,0.90$, and 0.93 at preintervention, post-intervention, post-visit, and 2, 6, and 12 weeks, respectively.

\section{Other measures}

Measures to assess variables that might influence or confound the relationship between adherence and pain severity

How often was each of the following statements true for you during the past 4 weeks?

1. I had a hard time doing what the doctor suggested I do.

2. I found it easy to do the things my doctor suggested I do.

3. I was unable to do what was necessary to follow my doctor's treatment plans.

4. I followed my doctor's suggestions exactly.

\section{Generally speaking, how often during the past 4 weeks were you able to do what the doctor told you?}

Figure I Medical Outcomes Study (MOS) general adherence measure items. Items 2, 4, and 5 were reverse coded. 
were also included. Basic demographic characteristics (age, race/ethnicity, sex, education level) were assessed using administrative records and the study screening and enrollment interviews. Participants were also asked at enrollment whether or not they were living with a partner. Cancer type (diagnosis) and stage were obtained via medical record review using a standardized medical record abstraction form. Mean inter-rater agreement (kappas) for abstraction of clinical data was 0.94 (range 0.84-1.0). Medical record abstractors also recorded the status of chemotherapy, hormonal therapy, radiotherapy, and surgical therapy for each patient. For surgical therapy, discrete status categories were scheduled to receive within 3 months, previously received, or none/status unknown. For the other three therapies, status categories were currently receiving/scheduled to begin within 2 weeks, previously received, or none/status unknown.

Health status was measured during the enrollment interview with the extensively validated MOS 12-item Short Form Health Survey (SF-12 ${ }^{\circledR}$ ) measure, from which Physical Component Summary (PCS-12) and Mental Component Summary (MCS-12) scores were derived. ${ }^{41-43}$ SF-12 items were scored and normalized via a standardized algorithm to calculate PCS-12 and MCS-12 scores ranging from 0 to 100 , with higher scores indicating better functioning. The MCS-12 and PCS-12 were designed to have mean scores of 50 and standard deviations of 10 in a representative sample of the US population. ${ }^{44}$

Finally, pain control self-efficacy was assessed at enrollment using three items drawn from the self-efficacy for pain management subscale of the Chronic Pain Self-Efficacy Scale. ${ }^{45}$ A 5-point Likert response scale was used for each item, from $1=$ not at all certain to $5=$ extremely certain; Cronbach's $\alpha$ in this sample was 0.67 .

\section{Statistical analysis}

Analyses were conducted using Stata (Version 11, StataCorp, College Station, TX, USA). Because of unmeasured influences, patient pain severity trajectories over time might be systematically underestimated or overestimated by our regression model, invalidating the important assumption that residual errors are independent. To account for this, we used mixed-effects regression models that included patientspecific varying (random) intercepts to assess the fixed effects of adherence and other independent variables on subsequent mean pain severity levels. ${ }^{46}$ The key dependent variables were pain severity at each of the four follow-up assessments (postvisit and 2, 6, and 12 weeks). The key independent variable was general adherence tendency at enrollment.
We employed a sequential modeling approach to explore the effects of adjusting for different groupings of covariates on the adherence/pain relationship. Model 1 included age category (10 year increments); gender; minority status (subjects self-identifying as other than non-Hispanic White [minority] versus those self-identifying as non-Hispanic White [non-minority], based on crossing responses to separate race and ethnicity questions); years of education $(<12,13-15$, and $>16)$; study group (experimental versus control); study site; baseline pain severity; and pain assessment time point. Model 2 included all Model 1 variables plus cancer type (lung, breast, prostate, head and neck, esophageal, colorectal, bladder, or gynecologic) and stage (metastatic or not), partner status (present versus absent), physical (PCS-12) and mental (MCS-12) health status, and pain control self-efficacy.

To address the directionality of the relationship between tendency to adhere and pain severity, we also performed a cross-lagged panel regression analysis by fitting two ordinary least squares (OLS) regression models and comparing the cross-lagged coefficients for adherence on pain severity. In the first OLS model, the dependent variable was pain severity at the 6 week follow-up and the independent variables were the same as in Model 1, yielding the cross-lagged regression coefficient for baseline adherence on 6 week pain severity. In the second OLS model, we replaced the dependent variable with the baseline pain severity score and replaced the independent variables for pain severity and adherence with the corresponding measures taken at the 6 week follow-up, yielding the cross-lagged regression coefficient for 6 week adherence on baseline pain severity. We compared these two regression coefficients using the method of Clogg et al. ${ }^{47}$ Supplementary and sensitivity analyses were also conducted to examine the effects of interactions between tendency to adhere and follow-up pain measurement time point, excluding baseline pain from the models, including adjustment for cancer treatment status (series of four dummy variables, one each for chemotherapy, hormonal therapy, radiotherapy, and surgical therapy), and removing variables from Model 2, one at a time, to consider their individual contributions.

\section{Results}

Of 3720 patients sent a recruitment letter, 3413 were excluded (1011 returned an opt-out postcard; 1015 could not be reached by telephone despite repeated calls; and 1387 were ineligible). The remaining 307 were randomized, and 265 received their randomly allocated intervention. Of these, 
224 had at least one follow-up pain severity assessment after the baseline assessment plus complete data for other Model 1 and 2 variables and were included in analyses.

Table 1 summarizes the characteristics of the current study sample. Subjects were predominantly women, nonminority, and well educated. Breast cancer and metastatic cancer affected over $50 \%$ of the sample. The mean baseline MOS general adherence score was high. The study sample had relatively poor mental and, in particular, physical health status, manifested by a mean MCS-12 score about half a standard deviation below the general US population mean, and a mean PCS-12 score about two standard deviations below the population mean. Mean pain severity was moderately high throughout the study but decreased during follow-up.

Table 2 shows the findings of the Model 1 analysis, adjusted only for basic sociodemographic characteristics, study group and site, pain assessment time point, and baseline pain. General tendency to adhere to provider-recommended treatments was significantly associated with pain severity over the 12 week follow-up period. A one standard deviation increase in the tendency to adhere to treatment was associated with a 0.22 -point adjusted mean decrease in pain severity ( $95 \%$ confidence interval [CI] 0.40, 0.03). Baseline pain was also significantly associated with follow-up pain severity. When baseline pain was excluded from Model 1, the association between tendency to adhere to treatment and subsequent pain was larger, such that a one standard deviation increase in tendency to adhere was associated with a 0.31 -point adjusted mean reduction in pain $(95 \% \mathrm{CI}$ $0.60,0.04)$. There was no significant interaction between adherence tendency and follow-up pain measurement time point $(P=0.64)$. Adjusting for cancer treatment status did not substantively change Model 1 findings (data not shown but available on request).

The cross-lagged model analysis revealed no significant association between baseline pain severity and tendency to adhere at 6 weeks (cross-lagged regression coefficient for 6 week adherence $=0.13,95 \% \mathrm{CI}=-0.07-0.32 ; P=0.21$ ), contrasting with the significant association observed between baseline tendency to adhere and 6 week pain severity (crosslagged regression coefficient for baseline adherence $=-0.29$, $95 \% \mathrm{CI}=-0.55$ to $-0.03 ; P=0.03)$. The regression coefficient for baseline tendency to adhere was significantly larger than that for tendency to adhere at 6 weeks (chi-square $=5.59$, degrees of freedom $=1, P=0.02$ ).

In the Model 2 analysis (Table 2), adjusting for all Model 1 variables plus characteristics that may influence
Table I Characteristics of study participants

\begin{tabular}{|c|c|}
\hline Characteristic & $N=224$ \\
\hline Age, years, mean (SD) & $58.7(9.5)$ \\
\hline Minority, \% & 28.1 \\
\hline Female, $\%$ & 77.7 \\
\hline \multicolumn{2}{|l|}{ Education, years completed, \% } \\
\hline$\leq 12$ & 29.0 \\
\hline $13-15$ & 29.9 \\
\hline$\geq 16$ & 41.1 \\
\hline Living with a partner, $\%$ & 62.1 \\
\hline Experimental group, \% & 50.9 \\
\hline \multicolumn{2}{|l|}{ Study site } \\
\hline Site I & 49.6 \\
\hline Site 2 & 47.8 \\
\hline Site 3 & 2.7 \\
\hline \multicolumn{2}{|l|}{ Cancer type, \% } \\
\hline Breast & 55.8 \\
\hline Lung & 18.3 \\
\hline Other & 25.9 \\
\hline Metastatic disease, \% & 56.3 \\
\hline \multicolumn{2}{|l|}{ Cancer treatment status, \% } \\
\hline \multicolumn{2}{|l|}{ Chemotherapy } \\
\hline Current or scheduled to begin within 2 weeks & 33.0 \\
\hline Previous & 51.3 \\
\hline None/unknown & 15.6 \\
\hline \multicolumn{2}{|l|}{ Hormonal therapy } \\
\hline Current or scheduled to begin within 2 weeks & 36.2 \\
\hline Previous & 10.3 \\
\hline None/unknown & 53.6 \\
\hline \multicolumn{2}{|l|}{ Radiotherapy } \\
\hline Current or scheduled to begin within 2 weeks & 8.0 \\
\hline Previous & 51.3 \\
\hline None/unknown & 40.6 \\
\hline \multicolumn{2}{|l|}{ Surgical therapy } \\
\hline Scheduled to receive within 3 months & 0.4 \\
\hline Previous & 81.7 \\
\hline None/unknown & 17.9 \\
\hline \multicolumn{2}{|l|}{ Health status, mean (SD) } \\
\hline Physical (PCS-I2) & $32.6(8.8)$ \\
\hline Mental (MCS-I2) & $44.3(10.1)$ \\
\hline Pain control self-efficacy, mean (SD) & $3.1(1.0)$ \\
\hline \multicolumn{2}{|l|}{ MOS General Adherence score, mean (SD) } \\
\hline Enrollment & $5.1(1.0)$ \\
\hline 6 weeks & $5.2(1.0)$ \\
\hline \multicolumn{2}{|l|}{ Pain severity, mean (SD) } \\
\hline Enrollment & $6.6(1.8)$ \\
\hline Pre-intervention & $6.4(2.0)$ \\
\hline Post-intervention & $6.3(2.0)$ \\
\hline Post-visit & $6.3(2.0)$ \\
\hline 2 weeks & $6.2(2.1)$ \\
\hline 6 weeks & $5.9(2.2)$ \\
\hline 12 weeks & $5.7(2.4)$ \\
\hline
\end{tabular}

Abbreviations: MCS-12, Short Form Health Survey Mental Component Summary; MOS, Medical Outcomes Study; PCS-12, Short Form Health Survey Physical Component Summary; SD, standard deviation. 
Table 2 Relationship between participant characteristics and follow-up pain severity

\begin{tabular}{|c|c|c|c|c|}
\hline Characteristic & 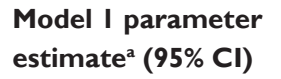 & $P$ value & 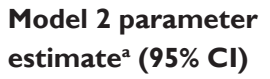 & $P$ value \\
\hline Adherence & $-0.20(-0.38,-0.03)$ & 0.02 & $-0.14(-0.31,0.04)$ & 0.12 \\
\hline Age & $0.06(-0.12,0.23)$ & 0.52 & $0.03(-0.14,0.20)$ & 0.72 \\
\hline Racial/ethnic minority & $0.05(-0.3 \mathrm{I}, 0.4 \mathrm{I})$ & 0.79 & $0.04(-0.30,0.39)$ & 0.81 \\
\hline Female & $0.12(-0.28,0.52)$ & 0.56 & $0.13(-0.34,0.61)$ & 0.58 \\
\hline \multicolumn{5}{|c|}{ Education (ref $=$ high school or less) } \\
\hline Some college & $0.18(-0.24,0.61)$ & 0.40 & $0.15(-0.26,0.56)$ & 0.47 \\
\hline College graduate & $-0.25(-0.65,0.15)$ & 0.21 & $-0.23(-0.62,0.16)$ & 0.25 \\
\hline Experimental group & $-0.10(-0.42,0.22)$ & 0.54 & $-0.04(-0.35,0.28)$ & 0.82 \\
\hline \multicolumn{5}{|l|}{ Study site $($ ref $=$ site I $)$} \\
\hline Site 2 & $-0.16(-0.49,0.16)$ & 0.32 & $-0.28(-0.59,0.04)$ & 0.09 \\
\hline Site 3 & $0.18(-0.82,1.18)$ & 0.72 & $0.42(-0.57,1.40)$ & 0.41 \\
\hline \multicolumn{5}{|c|}{ Pain measurement time point (ref = pre-intervention) } \\
\hline Post-intervention & $-0.11(-0.33,0.12)$ & 0.35 & $-0.11(-0.33,0.12)$ & 0.35 \\
\hline Post-visit & $-0.13(-0.35,0.09)$ & 0.26 & $-0.13(-0.35,0.09)$ & 0.26 \\
\hline 2 weeks & $-0.18(-0.40,0.05)$ & 0.12 & $-0.18(-0.41,0.05)$ & 0.12 \\
\hline 6 weeks & $-0.42(-0.65,-0.19)$ & $<0.01$ & $-0.42(-0.64,-0.19)$ & $<0.01$ \\
\hline 12 weeks & $-0.61(-0.84,-0.38)$ & $<0.01$ & $-0.61(-0.84,-0.38)$ & $<0.01$ \\
\hline Baseline pain severity & $0.73(0.64,0.82)$ & $<0.01$ & $0.64(0.54,0.73)$ & $<0.01$ \\
\hline \multicolumn{5}{|l|}{ Cancer type (ref = breast) } \\
\hline Lung & & & $-0.13(-0.59,0.33)$ & 0.57 \\
\hline All others & & & $-0.16(-0.62,0.30)$ & 0.51 \\
\hline Metastatic disease & & & $0.32(-0.03,0.67)$ & 0.07 \\
\hline Living with partner & & & $-0.18(-0.51,0.14)$ & 0.27 \\
\hline PCS- 12 & & & $-0.04(-0.06,-0.02)$ & $<0.01$ \\
\hline MCS-I2 & & & $-0.02(-0.03,-0.00)$ & 0.05 \\
\hline Pain control self-efficacy & & & $-0.01(-0.19,0.17)$ & 0.92 \\
\hline
\end{tabular}

Note: aparameter estimates are the estimated regression coefficients from a mixed effects multiple linear regression model.

Abbreviations: Cl, confidence interval; MCS-12, Short Form Health Survey Mental Component Summary; PCS-12, Short Form Health Survey Physical Component Summary; ref, analytic reference.

tendency to adhere and/or pain severity (partner status, cancer type and degree of spread, physical and mental health, and pain control self-efficacy), the association between tendency to adhere and follow-up pain severity was attenuated and no longer statistically significant. This remained true when baseline pain severity was removed from the model and when adjustment for cancer treatment status was included (data not shown but available on request).

In exploratory analyses removing covariates individually from Model 2, only the omission of PCS-12 or MCS-12 scores (and not partner status or pain control selfefficacy score) substantively increased the strength of the association between tendency to adhere and pain severity, changing the estimated regression coefficient for tendency to adhere from $-0.14(95 \% \mathrm{CI}-0.31,0.04, P=0.12)$ in the full model to $-0.18(95 \% \mathrm{CI}-0.36,-0.01, P=0.04)$ and -0.18 (95\% CI $-0.35,-0.01, P=0.04)$, respectively.

\section{Discussion}

The relationship between tendency to adhere to providerrecommended treatments and subsequent pain severity among cancer patients has not been previously examined. This is an important oversight, given the generally suboptimal state of cancer pain control, ${ }^{1,2}$ the high societal prevalence of serious misgivings about the safety of analgesics commonly prescribed to treat cancer pain, ${ }^{1,2,6,7}$ and the apparently low levels of adherence to recommended cancer pain treatment regimens. ${ }^{3,4}$ Our findings begin to address this important yet previously neglected relationship.

In a secondary observational analysis of prospective data from patients enrolled in an RCT of a cancer pain coaching intervention, we found patients' general tendency to adhere to provider-recommended treatments at baseline predicted cancer pain severity over 12 weeks' follow-up in a model adjusting for basic sociodemographic characteristics, RCT-related variables, and baseline pain (Model 1). 
The sociodemographic characteristics in this model (age, gender, race/ethnicity, and education) were included because each has been associated with pain ${ }^{15}$ and adherence ${ }^{22}$ in prior studies. Particularly in the context of the observed significant association between general tendency to adhere and pain severity, we considered that including baseline pain in the model, although conservative, might constitute overadjustment, as some of the influence of adherence tendency on pain severity would likely have been exerted at the start of the study. Consistent with this reasoning, in an analysis omitting baseline pain from Model 1, there was an additional 0.1-point decrement in adjusted mean pain severity associated with a one standard deviation increase in tendency to adhere. On the other hand, not adjusting for baseline pain may represent underadjustment. Thus, the "true" point estimate of the association between baseline general treatment adherence tendency and subsequent pain severity may lie somewhere between these values.

These findings are broadly consistent with those of a large number of prior studies employing a variety of adherence measures, including measures of general tendency to adhere, and involving patients with diseases other than cancer and outcomes other than pain severity. ${ }^{8}$ Additionally, a cross-lagged model analysis revealed no significant association between baseline pain severity and 6 week adherence tendency, contrasting with the aforementioned significant association between baseline adherence tendency and 6 week pain severity, and baseline tendency to adhere parameter estimate was significantly larger than the 6 week tendency to adhere parameter estimate. These findings suggest that a simple "halo effect" due to underlying patient dispositional characteristics influencing both adherence tendency and pain severity is unlikely to account for the observed association between adherence tendency and subsequent pain severity. Rather, the temporal sequencing of the relationship (baseline adherence tendency associated with subsequent pain, but baseline pain not associated with subsequent adherence tendency) is consistent with a causal relationship. Thus, measurement of baseline tendency to adhere to recommended treatments may be useful in predicting pain severity over time among cancer patients. Furthermore, studies involving individuals with noncancer health conditions suggest "tendency to adhere" is a more mutable characteristic than the label might imply, and so might represent a useful therapeutic target. Future RCTs of interventions expressly designed to improve cancer pain control through salutary effects on tendency to adhere would be helpful to more definitively investigate these possibilities.

To further explore the relationship between adherence tendency and pain severity, we also created an expanded model that included all Model 1 variables as well as several personal characteristics likely to influence the tendency to adhere, pain severity, or both: partner status, mental and physical health status, and pain control self-efficacy (Model 2). These additional variables were included in the model based on previously demonstrated associations with pain ${ }^{14,16-21}$ as well as with adherence. ${ }^{23-36}$ In this model, patient tendency to adhere was no longer significantly associated with follow-up pain severity, and the inclusion of two variables in particular (physical and mental health status) appeared to account for this finding. This is again generally consistent with prior research involving patients with health conditions other than cancer, in which lower mental health status ${ }^{28-33}$ and, particularly for more burdensome health conditions, lower physical health status ${ }^{34-36}$ have been associated with lower adherence estimates, including lower tendency to adhere as assessed with the MOS general adherence measure. ${ }^{33}$

One potential interpretation of this finding is that the general tendency to adhere to provider-recommended treatments influences subsequent pain severity among cancer patients, and that physical and mental health statuses mediate this effect. Individuals who assess themselves as being more likely to adhere to provider-recommended treatments may actually be more adherent to specific pain treatments (not measured in the current study). Better adherence could improve physical and mental health status, in turn leading to less "actual" pain (due to reduced physical illness burden) and reduced tendency to report severe pain (due to improved mental health and better coping). Alternatively, physical and mental health status may confound the relationship between tendency to adhere and subsequent cancer pain severity. Such confounding could reflect the influence of relatively fixed underlying dispositional characteristics (eg, optimism on the "positive" side and neuroticism on the "negative" side). Such characteristics are likely to influence patient responses to each of the relevant self-report measures (adherence tendency, health status, pain severity), which seek to capture respondent standing on conceptually different yet, in reality, partially overlapping constructs. Including the SF-12 in our models might also result in overadjustment, as the measure includes one item concerning pain (asking respondents to rate how much pain interfered with their normal work during the preceding 4 weeks). Another potential source 
of confounding is that individuals with poor health status, reflecting poor control of their health conditions, may also have more pain, which might impair their ability to adhere to recommended treatments. In the context of observational data such as those available here, the distinction between mediation and confounding cannot be determined, ${ }^{48}$ yet the research and clinical implications of each explanation would be somewhat different. Future studies will be required to clarify this issue.

Our study had a number of strengths, including a prospective design, the use of rigorous analytic methods, and a sample that included patients with a range of cancer types and severities. Our study also had some limitations. Though our analyses examined temporal associations between adherence and pain severity, causality cannot be inferred from these observational relationships. The study data were also drawn from a sample of English-speaking cancer patients cared for by cancer specialists in one metropolitan area, so the findings may or may not be generalize to other patients, physicians, or geographic regions. Finally, the parent RCT from which the study data were drawn included a single adherence measure assessing patients' global tendency to adhere to provider-recommended treatments. Thus, we were unable to examine the relationship between actual patient adherence to specific recommended pain treatments, such as individual prescription or over-the-counter pain medications, and pain severity. The findings of analyses similar to ours but that focus on the effects of adherence to specific recommended treatments might differ.

In conclusion, in a secondary analysis of data from an RCT of cancer patient coaching intervention, we found that baseline general patient tendency to adhere to providerrecommended treatments was significantly associated with cancer pain severity over 12 weeks when adjusting for basic sociodemographic characteristics, RCT-related variables, and baseline pain. Furthermore, the results of a cross-lagged analysis suggested this association is unlikely to merely reflect underlying patient dispositional characteristics that influence both adherence tendency and pain severity. If confirmed in other studies designed specifically to examine causality, this finding would suggest that general tendency to adhere to provider-recommended treatments might be useful as a predictor of subsequent cancer pain severity and as a target of interventions to improve cancer pain control. Tempering this finding somewhat, we also found that after further adjusting for health status, the association between adherence tendency and pain severity was no longer significant. Thus, health status either mediated or confounded the adherence tendency/pain severity relationship, with each of these potential interpretations having somewhat different research and clinical implications. Further prospective studies designed to clarify the nature of the relationships among tendency to adhere, health status, and pain severity among cancer patients appear warranted.

\section{Disclosure}

The authors report no conflicts of interest in this work.

\section{References}

1. Fairchild A. Under-treatment of cancer pain. Curr Opin Support Palliat Care. 2010;4(1):11-15.

2. Weiss SC, Emanuel LL, Fairclough DL, Emanuel EJ. Understanding the experience of pain in terminally ill patients. Lancet. 2001;357(9265):1311-1315.

3. Miaskowski C, Dodd MJ, West C, et al. Lack of adherence with the analgesic regimen: a significant barrier to effective cancer pain management. J Clin Oncol. 2001;19(23):4275-4279.

4. Valeberg BT, Miaskowski C, Hanestad BR, Bjordal K, Moum T, Rustoen T. Prevalence rates for and predictors of self-reported adherence of oncology outpatients with analgesic medications. Clin J Pain. 2008;24(7):627-636.

5. Davis MP, Walsh D. Epidemiology of cancer pain and factors influencing poor pain control. Am J Hosp Palliat Care. 2004;21(2): 137-142.

6. Ersek M, Kraybill BM, Pen AD. Factors hindering patients' use of medications for cancer pain. Cancer Pract. 1999;7(5):226-232.

7. Fitzcharles MA, DaCosta D, Ware MA, ShirY. Patient barriers to pain management may contribute to poor pain control in rheumatoid arthritis. J Pain. 2009;10(3):300-305.

8. DiMatteo MR, Giordani PJ, Lepper HS, Croghan TW. Patient adherence and medical treatment outcomes: a meta-analysis. Med Care. 2002; 40(9):794-811.

9. Haynes RB, Ackloo E, Sahota N, McDonald HP, Yao X. Interventions for enhancing medication adherence. Cochrane Database Syst Rev. 2008;16(2):CD000011.

10. Kravitz RL, Tancredi DJ, Street RL Jr, et al. Cancer Health Empowerment for Living without Pain (Ca-HELP): study design and rationale for a tailored education and coaching intervention to enhance care of cancer-related pain. BMC Cancer. 2009;9:319.

11. Street RL Jr, Slee C, Kalauokalani DK, Dean DE, Tancredi DJ, Kravitz RL. Improving physician-patient communication about cancer pain with a tailored education-coaching intervention. Patient Educ Couns. 2010;80(1):42-47.

12. Medical Outcomes Study (MOS) measures of patient adherence [homepage on the Internet]. Santa Monica, CA: RAND Corporation, c1994-2010 [updated 2009 Oct 26; cited 2010 Oct 20]. http://www. rand.org/health/surveys_tools $/ \mathrm{mos} / \mathrm{mos}$ _adherence.html. Accessed Jul 202010.

13. Sherbourne CD, Hays RD, Ordway L, DiMatteo MR, Kravitz RL. Antecedents of adherence to medical recommendations: results from the Medical Outcomes Study. J Behav Med. 1992;15(5):447-468.

14. Van den Beuken-van Everdingen MH, de Rijke JM, Kessels AG, Schouten HC, van Kleef M, Patijn J. Prevalence of pain in patients with cancer: a systematic review of the past 40 years. Ann Oncol. 2007;18(9): 1437-1449.

15. Green CR, Hart-Johnson T. Cancer pain: an age-based analysis. Pain Med. 2010;11(10):1525-1536.

16. Somers TJ, Shelby RA, Keefe FJ, et al. Disease severity and domainspecific arthritis self-efficacy: relationships to pain and functioning in patients with rheumatoid arthritis. Arthritis Care Res (Hoboken). 2010; 62(6):848-856. 
17. Katz J, Poleshuck EL, Andrus CH, et al. Risk factors for acute pain and its persistence following breast cancer surgery. Pain. 2005;119(1-3): 16-25.

18. Mosher CE, Duhamel KN, Egert J, Smith MY. Self-efficacy for coping with cancer in a multiethnic sample of breast cancer patients: associations with barriers to pain management and distress. Clin J Pain. 2010;26(3):227-234.

19. Blamey R, Jolly K, Greenfield S, Jobanputra P. Patterns of analgesic use, pain and self-efficacy: a cross-sectional study of patients attending a hospital rheumatology clinic. BMC Musculoskelet Disord. 2009;10:137.

20. Liang SY, Yates P, Edwards H, Tsay SL. Factors influencing opioidtaking self-efficacy and analgesic adherence in Taiwanese outpatients with cancer. Psychooncology. 2008;17(11):1100-1107.

21. Thomas E, Silman AJ, Croft PR, Papageorgiou AC, Jayson MI, Macfarlane GJ. Predicting who develops chronic low back pain in primary care: a prospective study. BMJ. 1999;318(7199):1662-1667.

22. DiMatteo MR. Variations in patients' adherence to medical recommendations: a quantitative review of 50 years of research. Med Care. 2004;42(3):200-209.

23. DiMatteo MR, Lepper HS, Croghan TW. Depression is a risk factor for noncompliance with medical treatment: meta-analysis of the effects of anxiety and depression on patient adherence. Arch Intern Med. 2000; 160(14):2101-2107.

24. Sayers SL, Riegel B, Pawlowski S, Coyne JC, Samaha FF. Social support and self-care of patients with heart failure. Ann Behav Med. 2008; 35(1):70-79.

25. Holt EW, Muntner P, Joyce CJ, Webber L, Krousel-Wood MA. Healthrelated quality of life and antihypertensive medication adherence among older adults. Age Ageing. 2010;39(4):481-487.

26. Chen HF, Tsai YF, Lin YP, Shih MS, Chen JC. The relationships among medicine symptom distress, self-efficacy, patient-provider relationship, and medication compliance in patients with epilepsy. Epilepsy Behav. 2010;19(1):43-49.

27. Schousboe JT, Dowd BE, Davison ML, Kane RL. Association of medication attitudes with non-persistence and non-compliance with medication to prevent fractures. Osteoporos Int. 2010;21(11):1899-1909.

28. Trivedi RB, Ayotte B, Edelman D, Bosworth HB. The association of emotional well-being and marital status with treatment adherence among patients with hypertension. J Behav Med. 2008;31(6):489-497.

29. Viswanathan H, Anderson R, Thomas J 3rd. Nature and correlates of SF-12 physical and mental quality of life components among lowincome HIV adults using an HIV service center. Qual Life Res. 2005; 14(4):935-944.

30. Platt AB, Localio AR, Brensinger CM, et al. Risk factors for nonadherence to warfarin: results from the IN-RANGE study. Pharmacoepidemiol Drug Saf. 2008;17(9):853-860.

31. Royal SW, Kidder DP, Patrabansh S, et al. Factors associated with adherence to highly active antiretroviral therapy in homeless or unstably housed adults living with HIV. AIDS Care. 2009;21(4):448-455.

32. Unson CG, Litt M, Reisine S, Mahoney-Trella P, Sheperd T, Prestwood K Adherence to calcium/vitamin D and estrogen protocols among diverse older participants enrolled in a clinical trial. Contemp Clin Trials. 2006;27(3):215-226.
33. Jerant A, DiMatteo R, Arnsten J, Moore-Hill M, Franks P. Self-report adherence measures in chronic illness: retest reliability and predictive validity. Med Care. 2008;46(11):1134-1139.

34. Bernstein D, Kleinman L, Barker CM, Revicki DA, Green J. Relationship of health-related quality of life to treatment adherence and sustained response in chronic hepatitis C patients. Hepatology. 2002; 35(3):704-708.

35. Cote I, Farris K, Feeny D. Is adherence to drug treatment correlated with health-related quality of life? Qual Life Res. 2003;12(6): 621-633.

36. Pavone RM, Burnett KF, LaPerriere A, Perna FM. Social cognitive and physical health determinants of exercise adherence for HIV-1 seropositive, early symptomatic men and women. Int J Behav Med. 1998;5(3):245-258.

37. Hamilton GA. Measuring adherence in a hypertension clinical trial. Eur J Cardiovasc Nurs. 2003;2(3):219-228.

38. Zugelj U, Zupancic M, Komidar L, Kenda R, Varda NM, Gregoric A Self-reported adherence behavior in adolescent hypertensive patients: the role of illness representations and personality. J Pediatr Psychol. 2010;35(9):1049-1060.

39. Holstad MM, Foster V, Diiorio C, McCarty F, Teplinskiy I. An examination of the psychometric properties of the Antiretroviral General Adherence Scale (AGAS) in two samples of HIV-infected individuals. J Assoc Nurses AIDS Care. 2010;21(2):162-172.

40. McDonnell M, Turner J, Weaver MT. Antecedents of adherence to antituberculosis therapy. Public Health Nurs. 2001;18(6):392-400.

41. McHorney CA, Ware JE Jr, Raczek AE. The MOS 36-item short-form health survey (SF-36): II. psychometric and clinical tests of validity in measuring physical and mental health constructs. Med Care. 1993; 31(3):247-263.

42. Ware J Jr, Kosinski M, Keller SD. A 12-item short-form health survey: construction of scales and preliminary tests of reliability and validity. Med Care. 1996;34(3):220-233.

43. Ware JE Jr, Kosinski M, Bayliss MS, McHorney CA, Rogers WH, Raczek A. Comparison of methods for the scoring and statistical analysis of SF-36 health profile and summary measures: summary of results from the Medical Outcomes Study. Med Care. 1995;33 Suppl 4: AS264-AS279.

44. Ware JE Jr, Kosinski M, Keller SD. How to score the SF-12 Physical and Mental Health Summary Scales. 3rd ed. Lincoln, RI: QualityMetric, Inc; 1998.

45. Anderson KO, Dowds BN, Pelletz RE, Edwards WT, PeetersAsdourian C. Development and initial validation of a scale to measure self-efficacy beliefs in patients with chronic pain. Pain. 1995;63(1) 77-84.

46. Gelman A, Hill J. Data Analysis using Regression and Multilevel/ Hierarchical Models. New York: Cambridge University Press; 2007.

47. Clogg CC, Petkova E, Haritou A. Statistical methods for comparing regression coefficients between models. Am J Sociol. 1995; 100(5):1261-1293.

48. MacKinnon DP, Krull JL, Lockwood CM. Equivalence of the mediation, confounding and suppression effect. Prev Sci. 2000;1(4): 173-181.
Patient Preference and Adherence

\section{Publish your work in this journal}

Patient Preference and Adherence is an international, peer-reviewed, open access journal focusing on the growing importance of patient preference and adherence throughout the therapeutic continuum. Patient satisfaction, acceptability, quality of life, compliance, persistence and their role in developing new therapeutic modalities and compounds to

\section{Dovepress}

optimize clinical outcomes for existing disease states are major areas of interest. This journal has been accepted for indexing on PubMed Central. The manuscript management system is completely online and includes a very quick and fair peer-review system. Visit http://www.dovepress.com/ testimonials.php to read real quotes from published authors. 